\title{
Supernova-driven interstellar turbulence and the galactic dynamo
}

\author{
Oliver Gressel $^{1}$, Detlef Elstner ${ }^{2}$ \& Günther Rüdiger ${ }^{2}$ \\ ${ }^{1}$ Astronomy Unit, Queen Mary, University of London, Mile End Road, London E1 4NS, UK \\ ${ }^{2}$ Astrophysikalisches Institut Potsdam, An der Sternwarte 16, 14482 Potsdam, Germany \\ email: o.gressel@qmul.ac.uk, elstner@aip.de, gruediger@aip.de
}

\begin{abstract}
The fractal shape and multi-component nature of the interstellar medium together with its vast range of dynamical scales provides one of the great challenges in theoretical and numerical astrophysics. Here we will review recent progress in the direct modelling of interstellar hydromagnetic turbulence, focusing on the role of energy injection by supernova explosions. The implications for dynamo theory will be discussed in the context of the mean-field approach.

Results obtained with the test field-method are confronted with analytical predictions and estimates from quasilinear theory. The simulation results enforce the classical understanding of a turbulent Galactic dynamo and, more importantly, yield new quantitative insights. The derived scaling relations enable confident global mean-field modelling.
\end{abstract}

Keywords. turbulence - ISM: supernova remnants, dynamics, magnetic fields

\section{Interstellar turbulence}

Apart from stars, the baryonic matter within the Galaxy is in the form of an extremely dilute, turbulent plasma known as the interstellar medium (ISM). The multitude of physical processes within the ISM entails a rich heterogeneous structure (Spitzer, 1978).

Approximating radiative processes by a simplified cooling prescription, and restricting the computational domain to a local patch, the turbulent ISM is now routinely modelled by means of three-dimensional fluid simulations (Korpi et al., 1999; Melioli \& de Gouveia Dal Pino, 2004; Slyz et al., 2005; Joung \& Mac Low, 2006; Dib et al., 2006). One main focus of these simulations has been to obtain filling factors of the different ISM phases and compare them to the classical predictions as well as observations (Dettmar, 1992). Further topics of interest include turbulent mixing (Balsara \& Kim, 2005), thermodynamic distribution functions (Mac Low et al., 2005), and line-of-sight integrated column densities (de Avillez \& Breitschwerdt, 2005b).

\subsection{The small-scale dynamo}

While various simulations (Korpi et al., 1999b; de Avillez \& Breitschwerdt, 2005a; Mac Low et al., 2005) discuss the influence of magnetic fields on the ISM morphology, little is said about the actual mechanism of field amplification. Balsara et al. (2004) have addressed this question by means of unstratified simulations of SNe turbulence. The authors relate the growth of small-scale magnetic fields to vorticity production in supernova shocks (Balsara et al., 2001), and chaotic field line-stretching (Balsara \& Kim, 2005).

The fact that vorticity production by colliding shells is almost inevitable in a clumpy and highly structured ISM has first been pointed out by Korpi et al. (1999b). The issue has then been investigated for the simplified case of driven expansion waves by Mee \& Brandenburg (2006) and, more recently, by Del Sordo \& Brandenburg (this volume). Considering turbulence driven by non-helical transverse waves, Haugen et al., (2004) have 
shown that the small-scale dynamo becomes harder to excite in the super-sonic regime, albeit the critical Reynolds number for the onset of dynamo action only seems to depend weakly on the Mach number.

Because the eddy turnover time is short at small scales, a dynamo based on chaotic field line stretching will be fast. This is in-line with observations see and this volume, which exhibit dominant turbulent fields. Open issues remain with respect to the mechanism governing the saturation of the small-scale dynamo. Therefore, it is currently unclear whether equipartition field strengths can be obtained by a non-helical dynamo alone. Alternatively, the turbulent field might be explained as a "shredded" coherent field, i.e., as the by-product of a helical mean-field dynamo.

\section{The large-scale galactic dynamo}

Notwithstanding the above, the presence of the observed coherent fields on scales larger than the outer scale of the interstellar turbulence $(\sim 50 \mathrm{pc}$, see Fletcher et al., 2010) clearly requires the presence of a coherent dynamo. The favoured candidates for such a dynamo are, in no particular order: (i) the kinetic driving by SNe (Korpi et al., 1999; Gressel et al., 2008b; Gissinger et al., 2009) (ii) the buoyant cosmic ray-supported Parker instability (Parker, 1992; Hanasz et al., 2004), (iii) the magneto-rotational instability (Sellwood \& Balbus, 1999; Dziourkevitch et al., 2004; Nishikori et al., 2006; Piontek \& Ostriker, 2007), and (iv) gravitational interactions (Kotarba et al., 2010). $\dagger$

The last of these effects is certainly dominant at the early stages of galaxy formation and will provide a seed field for subsequent processes. It remains to be shown, however, how important external interactions are in the presence of a realistic feedback from scales currently unresolved in cosmological simulations. Moreover, as Hanasz et al., (2009) have shown, the cosmic ray (CR) dynamo critically relies on the anisotropy of the CR diffusion coefficient. Combined simulations, including both SNe and CRs, will prove whether this anisotropy remains effective for strongly tangled turbulent fields.

The MRI will be important (at least) in the outer regions of galaxies, where the star formation activity is low (Korpi et al. 2010) - even under moderate turbulence, it may operate efficiently. The stability criterion for a global isothermal disk of thickness $2 \mathrm{H}$ leads to the relation

$$
8 \sqrt{\mathrm{Pm}_{\mathrm{t}}}<C_{\Omega} \equiv \frac{\Omega H^{2}}{\eta_{\mathrm{t}}}
$$

for instability (Kitchatinov \& Rüdiger, 2004). Depending on the local rotation frequency, $\Omega$, turbulent diffusivity $\eta_{\mathrm{t}}$, and turbulent magnetic Prandtl number $\mathrm{Pm}_{\mathrm{t}}-$ which is generally expected to be of order unity (Fromang \& Stone, 2009) - this value may be lower than the critical dynamo number for the supernova-driven dynamo. It should be worthwhile to address this question within state-of-the-art simulations of the ISM, with an Alvfén velocity of the external vertical field of the order $1 \mathrm{~km} \mathrm{~s}^{-1}$.

\subsection{The supernova-driven dynamo}

The energy input through SNe into the ISM is tremendous. The corresponding amplitude of the expected dynamo effect has first been estimated by Sokoloff \& Shukurov (1990). Assuming hydrostatic equilibrium and applying quasi-linear theory (Rüdiger \& Kitchatinov, 1993), these estimates have subsequently been refined by (Fröhlich \& Schultz, 1996). A shortcoming of the approach was the neglect of a possible galactic wind. In general,

$\dagger$ Also see the respective reviews by Hanasz, Otmianowska-Mazur, and Lesch (this volume). 

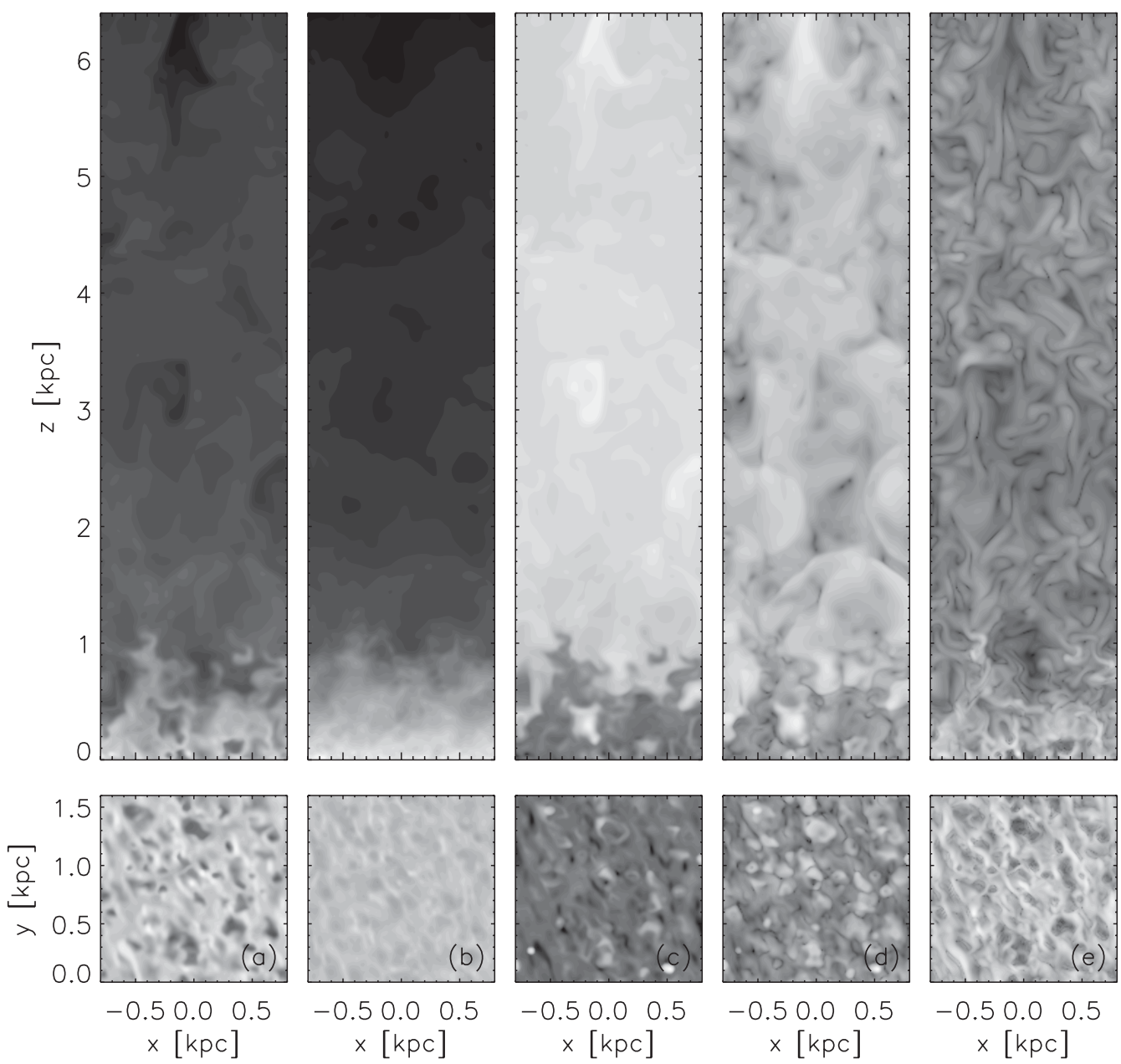

Figure 1. Snapshots at time $t=72 \mathrm{Myr}$ of the top half of the now larger simulation box (upper panels), and the disc midplane (lower panels). The variables shown are: (a) number density $\left[\mathrm{cm}^{-3}\right]$, (b) column density $\left[\mathrm{cm}^{-2}\right]$, (c) temperature $[\mathrm{K}]$, (d) velocity dispersion $\left[\mathrm{km} \mathrm{s}^{-1}\right]$, and (e) magnetic field strength $[\mu G]$. The logarithmic grey scales cover ranges $[-4.76,1.01]$, $[17.56,21.83],[2.13,7.03],[-0.61,2.64]$, and $[-5.98,-1.18]$, respectively.

there was a controversy as to whether the turbulence created by the SNe was too vigorous to warrant dynamo action - either because of a too strong wind (carrying the field away), or contrary to this, because of a too strong downward pumping (enhancing turbulent dissipation near the midplane).

In a series of papers, (Ferrière, 1998) analytically derived the electromotive force stemming from isolated remnants; the line of work was also later supported by simulations of single remnants (Kaisig et al., 1993; Ziegler et al. 1996). To obtain the net $\alpha$ effect, a convolution with an assumed vertical SN distribution was applied. However, the approach suffered from a too weak dynamo and highly dominant (upward) pumping. Only when considering the stratified nature of the galactic disc (Ferrière, 1998), the issue was somewhat alleviated, although still predicting a strong upward pumping.

Pioneering semi-global simulations based on "first principles" where performed by Korpi et al., (1999), and it was only for the fact of a too low dynamo number that no field amplification was observed in their simulations. The first direct simulations exhibiting 

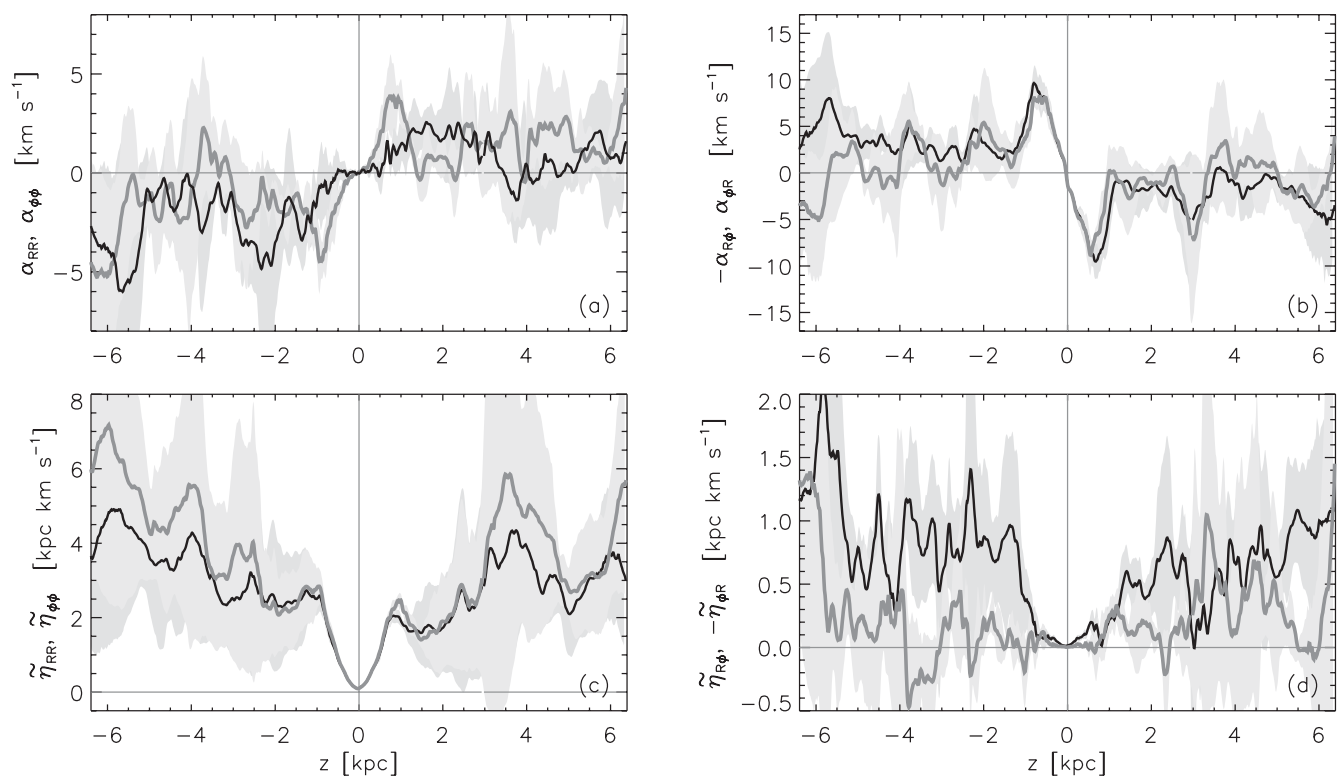

Figure 2. Dynamo coefficients as a function of the height $z$. The Variables are plotted in dark $\left(\alpha_{R R}, \ldots\right)$ or light $\left(\alpha_{\phi \phi}, \ldots\right)$ colours, respectively. Shaded areas indicate $1 \sigma$-fluctuations. In the region for $|z|<2 \mathrm{kpc}$, the results agree well with a previous run applying a much smaller box.

dynamo action were reported almost a decade later (Gressel et al., 2008b). The general morphology of such simulations is illustrated in Figure 1; note the apparent correlation between the density (a) and magnetic field amplitude (e) near the midplane, indicating field amplification via compression. In contrast, away from the midplane (i.e., in the diffuse medium) the field shows more folded structures.

The fast growth of the dynamo can be understood rigorously in terms of mean-field theory, i.e. via a Reynolds-averaged induction equation

$$
\partial_{t} \overline{\mathbf{B}}=\nabla \times \overline{\mathcal{E}}+\nabla \times[\overline{\mathbf{u}} \times \overline{\mathbf{B}}-\eta \nabla \times \overline{\mathbf{B}}],
$$

and assuming a standard parametrisation

$$
\overline{\mathcal{E}}_{i}=\alpha_{i j} \bar{B}_{j}-\tilde{\eta}_{i j} \varepsilon_{j k l} \partial_{k} \bar{B}_{l}, \quad i, j \in\{R, \phi\}, k=z,
$$

with tensorial coefficients $\alpha_{i j}$ and $\tilde{\eta}_{i j}$. The determination of these closure parameters (Gressel et al., 2008a) hugely benefited from the development of the so-called test field method (Schrinner et al., 2005). For kinematically forced turbulence, this method allows to determine unambiguously all eight tensor components - and, in fact, none of them can be ignored (Gressel, 2009). Notably, we find a significant positive Rädler effect in the off-diagonal elements of the diffusivity tensor (see panel 'd' in Figure 2).

The measured $\alpha$ effect and turbulent diffusion are of the expected sign and magnitude (see Figure 2, panels ' $a$ ' and ' $c$ '). The key finding is that of a moderate downward pumping (panel 'b') as predicted by Rüdiger \& Kitchatinov (1993). This inward pumping has profound implications in compensating the effect of the equally strong upward mean flow (not shown), thus yielding ideal conditions for the dynamo. Moreover, a wind may aid the shedding of small-scale magnetic helicity as discussed in Sur et al., (2007). 


\section{Recent advances}

One major concern with the local box approximation was the issue of its limited dimension in the horizontal direction. In the low-pressure ambient medium of the galactic halo, supernova remnants can easily expand to several hundred parsec in diameter. Because of the periodic boundaries and the finite domain size, this leads to (spuriously) correlated self-interactions. The simulations of Korpi et al., (1999) used $L_{x}=L_{y}=500$ pc, which was justified for the limited vertical extent of their model. For our standard runs we applied $L_{x}=L_{y}=800 \mathrm{pc}$ together with a vertical box size of $\pm 2 \mathrm{kpc}$.

\subsection{Large box simulations}

Even with near kpc horizontal box size, the issue of self-interaction was still seen in the far halo region. To eliminate the possibility of an artificial dependence of the results on the horizontal box size, we carried out a fiducial simulation run at $L_{x}=1.6 \mathrm{kpc}$ (cf. Figures $1 \& 2$ ). In the region of overlap, the extended profiles agree well with their counterparts derived from the smaller boxes (cf. Fig. 4.5, model 'F4' in Gressel, 2009).

Beyond $\pm 2 \mathrm{kpc}$, the velocity dispersion increases significantly, enhancing the turbulent diffusion and resulting in noisy profiles for the other coefficients. Note, however, the pronounced systematic $\alpha$ effect in the region of strong vertical gradients near the midplane (panels 'a' and 'b' in Figure 2). This clearly supports the paradigm of helicity production being the direct result of inhomogeneous turbulence (Rüdiger \& Kitchatinov, 1993).

\subsection{Scaling relations}

Beyond the purpose of mere diagnostics, the profiles shown in Figure 2 serve as a foundation for global large eddy-simulations solving (2.2). We believe that the semi-global approach of vertically stratified local boxes captures the essential physics behind the turbulent $\alpha$ effect in the galactic disc. At the same time, we are aware that the local geometry implies certain restrictions on the permitted dynamo modes. Identifying global symmetries is however necessary for a direct comparison with observations.

To warrant global mean-field modelling of the $\alpha \Omega$ dynamo, the scaling of the measured closure coefficients with the relevant input parameters has to be obtained. This is important because the angular velocity $\Omega$, the local shear rate $q$, the supernova rate $\sigma$, and the midplane density $\rho_{\mathrm{c}}$ are functions of the galactocentric distance (Ferrière, 2001). Gressel et al., (2009) already made a first step in this direction by inferring the dependence on the supernova rate. Corresponding mean-field models have been reported in (Elstner et al., 2009). Further scaling relations with respect to $\Omega$ and $\rho_{\text {c }}$ will be reported elsewhere.

First estimates (subject to small number statistics) for a range of $0.1-1$ times the galactic star formation rate lead to the relations

$$
C_{\alpha} \sim \Omega H, \quad \text { and } \quad C_{\Omega} \sim \Omega^{1.5} H^{2} \sigma^{-0.5} .
$$

This has the consequence that the dynamo number $D \equiv C_{\alpha} C_{\Omega} \sim \Omega^{2.5} H^{3} \sigma^{-0.5}$ scales inversely with the star formation rate. Therefore we would not expect a stronger amplification of the large-scale field for stronger star formation activity. Lastly, the pitch angle $p \simeq \sqrt{C_{\alpha} / C_{\Omega}} \sim \Omega^{-0.25} H^{-0.5} \sigma^{0.25}$, in the kinematic regime, only seems to depend weakly on the studied parameters. The strongest dependence here is, however, expected from the local shear rate $q \equiv \mathrm{d} \ln \Omega / \mathrm{d} \ln R$, suggesting a dedicated parameter survey. 


\section{Conclusions and prospects}

Because current numerical simulations are limited to very moderate Reynolds numbers, it is important to understand how efficient the observed mechanisms remain under realistic conditions. To achieve this, it has proven fruitful to study simplified scenarios and run multiple parameter sets (see, for a comprehensive review Brandenburg \& Subramanian, 2005). Fortunately, the emerging physical effects are dominated by the outer scale of the turbulence, i.e., as soon as a rudimentary scale separation is achieved, the turbulent quantities should become independent of the actual micro scale.

The growing complexity of models challenges the distribution of computing time: increasing physical realism leaves little margin for the variation of key parameters, let alone convergence checks or running multiple representations of a single parameter set. Dedicated studies remain mandatory to segregate artificial trends from genuinely physical ones (Hanasz et al., 2009; Gressel et al., 2009).

The dilemma becomes even more apparent when looking at the recent trend to performing "resolved" global simulations. For these, convergence checks are a rare exception. While an "enhanced" diffusivity may be sufficiently approximated by the numerical truncation error on the grid scale, the diamagnetic pumping term certainly is not. Yet vertical transport has profound implications on the emerging dynamo modes and growth rates (Bardou et al., 2001).

In conclusion, we advocate a strategy that has been applied with great success in the design of aircraft, namely the concept of large eddy (or mean-field) simulations. Global fluid simulations currently cannot guarantee scale separation for all relevant physical scales. To obtain quantitatively correct results, we therefore believe that a sub-grid scale model is inevitable. Present local box simulations are a valuable means to provide a rigorous framework for the calibration of such a model.

\section{Acknowledgements}

This work used the NIRVANA code version 3.3 developed by Udo Ziegler at the Astrophysical Institute Potsdam. Computations were performed at the AIP babel cluster.

\section{References}

Balsara D., Benjamin R. A., \& Cox D. P., 2001, The Astrophysical Journal, 563, 800

Balsara D. S. \& Kim J., 2005, The Astrophysical Journal, 634, 390

Balsara D. S., Kim J., Mac Low M.-M., \& Mathews G. J., 2004, The Astrophysical Journal, 617, 339

Bardou A., von Rekowski B., Dobler W., Brandenburg A., \& Shukurov A., 2001, Astronomy $\&$ Astrophysics, 370, 635

Beck R., Brandenburg A., Moss D., Shukurov A., \& Sokoloff D., 1996, Ann. Rev. Astron. Ė Astroph., 34, 155

Brandenburg A. \& Subramanian K., 2005, Phys. Rep., 417, 1

de Avillez M. A. \& Breitschwerdt D., 2005a, Astronomy \&3 Astrophysics, 436, 585

de Avillez M. A. \& Breitschwerdt D., 2005b, The Astrophysical Journal, 634, L65

Dettmar R. J., 1992, Fundamentals of Cosmic Physics, 15, 143

Dib S., Bell E., \& Burkert A., 2006, The Astrophysical Journal, 638, 797

Dziourkevitch N., Elstner D., \& Rüdiger G., 2004, Astronomy \&3 Astrophysics, 423, L29

Elstner D., Gressel O., \& Rüdiger G., 2009, in IAU Symposium Vol. 259, pp 467-478

Ferrière K., 1998, Astronomy \& Astrophysics, 335, 488

Ferrière K. M., 2001, RMP, 73, 1031

Fletcher A., Beck R., Shukurov A., Berkhuijsen E. M., \& Horellou C., 2010, (astro-ph:1001.5230)

Fröhlich H.-E. \& Schultz M., 1996, Astronomy \& Astrophysics, 311, 451 
Fromang S. \& Stone J. M., 2009, Astronomy \& Astrophysics, 507, 19

Gissinger C., Fromang S., \& Dormy E., 2009, Monthly Notices of the Royal Astronomical Society, 394, L84

Gressel O., 2009, PhD thesis, University of Potsdam (astro-ph:1001.5187)

Gressel O., Ziegler U., Elstner D., \& Rüdiger G., 2008, AN, 329, 619

Gressel O., Elstner D., Ziegler U., \& Rüdiger G., 2008, Astronomy \& Astrophysics, 486, L35

Gressel O., Ziegler U., Elstner D., \& Rüdiger G., 2009, in IAU Symposium Vol. 259, pp 81-86

Hanasz M., Kowal G., Otmianowska-Mazur K., \& Lesch H., 2004, The Astrophysical Journal, 605, L33

Hanasz M., Otmianowska-Mazur K., Kowal G., \& Lesch H., 2009, Astronomy \& Astrophysics, 498,335

Haugen N. E. L., Brandenburg A., \& Mee A. J., 2004, Monthly Notices of the Royal Astronomical Society, 353, 947

Joung M. K. R. \& Mac Low M.-M., 2006, The Astrophysical Journal, 653, 1266

Kaisig M., Rüdiger G., \& Yorke H. W., 1993, Astronomy \&3 Astrophysics, 274, 757

Kitchatinov L. L. \& Rüdiger G., 2004, Astronomy \&5 Astrophysics, 424, 565

Korpi M. J., Brandenburg A., Shukurov A., \& Tuominen I., 1999b, Astronomy \& Astrophysics, 350,230

Korpi M. J., Brandenburg A., Shukurov A., Tuominen I., \& Nordlund Å., 1999, The Astrophysical Journal, 514, L99

Korpi M. J., Käpylä P. J., \& Väisälä M. S., 2010, Astronomische Nachrichten, 331, 34

Kotarba H., Karl S. J., Naab T., Johansson P. H., Dolag K., Lesch H., \& Stasyszyn F. A., 2010, The Astrophysical Journal, 716, 1438

Mac Low M.-M., Balsara D. S., Kim J., \& de Avillez M. A., 2005, The Astrophysical Journal, 626,864

Mee A. J. \& Brandenburg A., 2006, Monthly Notices of the Royal Astronomical Society, 370, 415

Melioli C. \& de Gouveia Dal Pino E. M., 2004, Astronomy \& Astrophysics, 424, 817

Nishikori H., Machida M. \& Matsumoto R., 2006, The Astrophysical Journal, 641, 862

Parker E. N., 1992, The Astrophysical Journal, 401, 137

Piontek R. A. \& Ostriker E. C., 2007, The Astrophysical Journal, 663, 183

Rüdiger G. \& Kitchatinov L. L., 1993, Astronomy \& Astrophysics, 269, 581

Schrinner M., Rädler K.-H., Schmitt D., Rheinhardt M., \& Christensen U., 2005, AN, 326, 245

Sellwood J. A. \& Balbus S. A., 1999, The Astrophysical Journal, 511, 660

Slyz A. D., Devriendt J. E. G., Bryan G., \& Silk J., 2005, Monthly Notices of the Royal Astronomical Society, 356, 737

Sokoloff D. \& Shukurov A., 1990, Nat., 347, 51

Spitzer L., 1978, Physical processes in the ISM. New York Wiley-Interscience, 1978. 333 p.

Sur S., Shukurov A. \& Subramanian K., 2007, Monthly Notices of the Royal Astronomical Society, 377,874

Ziegler U., Yorke H. W., \& Kaisig M., 1996, Astronomy \&J Astrophysics, 305, 114 